\title{
On a model semilinear elliptic equation in the plane
}

\author{
Vladimir Gutlyanskĭ, Olga Nesmelova, Vladimir Ryazanov
}

\begin{abstract}
Assume that $\Omega$ is a regular domain in the complex plane $\mathbb{C}$ and $A(z)$ is symmetric $2 \times 2$ matrix with measurable entries, $\operatorname{det} A=1$ and such that $1 / K|\xi|^{2} \leq\langle A(z) \xi, \xi\rangle \leq K|\xi|^{2}, \xi \in \mathbb{R}^{2}, 1 \leq K<$ $\infty$. We study the blow-up problem for a model semilinear equation $\operatorname{div}(A(z) \nabla u)=e^{u}$ in $\Omega$ and show that the well-known Liouville-Bieberbach function solves the problem under an appropriate choice of the matrix $A(z)$. The proof is based on the fact that every regular solution $u$ can be expressed as $u(z)=T(\omega(z))$ where $\omega: \Omega \rightarrow G$ stands for quasiconformal homeomorphism generated by the matrix $A(z)$ and $T$ is a solution of the semilinear weihted Bieberbach equation $\triangle T=m(w) e^{T}$ in $G$. Here the weight $m(w)$ is the Jacobian determinant of the inverse mapping $\omega^{-1}(w)$.
\end{abstract}

2010 MSC. 30C62, 35J61.

Key words and phrases. Semilinear elliptic equations, Bieberbach equation, quasiconformal mappings, Beltrami equation, Keller-Osserman condition.

\section{Introduction}

Let $\Omega$ be a simply connected domain in the complex plane $\mathbb{C}$. Denote by $M^{2 \times 2}(\mathbb{C})$ the class of two by two symmetric matrices $A(z)=\left\{a_{j k}\right\}$ with measurable entries and $\operatorname{det} A(z)=1$, satisfying the uniform ellipticity condition

$$
\frac{1}{K}|\xi|^{2} \leq a_{j k}(z) \xi_{i} \xi_{j} \leq K|\xi|^{2} \text { a.e. in } \Omega
$$

for every $\xi \in \mathbb{C}$. The factor $K$ here can be either a constant or a measurable function on $\Omega$ with $1 \leq K(z)<\infty$ almost everywhere in $\Omega$.

Given a matrix $A \in M^{2 \times 2}(\Omega)$, let us consider the following second order elliptic equation in divergence form

$$
\operatorname{div}(A(z) \nabla u)=0 \text { a.e in } \Omega .
$$

Received 09.03.2016 
Equation (1.2) is interpreted, generally speaking, in the distributional sense. That is, a function $u$ is a weak solution to the equation if it has locally integrable gradient $\nabla u$ with

$$
\int_{\Omega}\langle A(z) \nabla u, \nabla \varphi\rangle=0 \quad \forall \varphi \in C_{0}^{\infty}(\Omega) .
$$

This is meaningful at least for $u \in W_{\text {loc }}^{1,1}(\Omega)$. Dealing in the main with quasiconformal mappings, generated by the uniformly elliptic matrix $A \in$ $M^{2 \times 2}(\mathbb{C})$, one can assume a little more regularity, namely $u \in W_{\text {loc }}^{1,2}(\Omega)$. However, if we are going to study semilinear degenerate elliptic equation, then will assume under the weak solution $u \in W_{\text {loc }}^{1,1}(\Omega)$.

Let $A \in M^{2 \times 2}(\mathbb{C})$ and $u \in W_{\text {loc }}^{1,2}(\Omega)$ be a weak solution to (1.2). Then there exists $v \in W_{\text {loc }}^{1,2}(\Omega)$, called the stream function of $u$, such that one has

$$
\nabla v=S A \nabla u \text { a.e in } \Omega, S=\left(\begin{array}{rr}
0 & -1 \\
1 & 0
\end{array}\right) .
$$

Setting $\omega(z)=u+i v$, one writes in complex notations that $\omega$ satisfies the Beltrami equation

$$
\omega_{\bar{z}}(z)=\mu(z) \omega_{z}(z) \text { a.e. in } \Omega,
$$

where the complex dilatation $\mu(z)$ is given by

$$
\mu(z)=\frac{1}{\operatorname{det}(I+A)}\left(a_{22}-a_{11}-2 i a_{12}\right) .
$$

Here $I$ stands for the unit matrix.

The condition of ellipticity (1.1) now is written as

$$
|\mu(z)| \leq \frac{K-1}{K+1} \quad \text { a.e. in } \Omega .
$$

And vice versa, given a complex-valued function $\mu$, satisfying (1.7), one can invert the algebraic system (1.6) to obtain

$$
A(z)=\left(\begin{array}{cc}
\frac{|1-\mu|^{2}}{1-|\mu|^{2}} & \frac{-2 \operatorname{Im} \mu}{1-|\mu|^{2}} \\
\frac{-2 \mathrm{I} m \mu}{1-|\mu|^{2}} & \frac{|1+\mu|^{2}}{1-|\mu|^{2}}
\end{array}\right) .
$$

Thus, given any $A \in M^{2 \times 2}(\Omega)$, one produces by (1.6) the complex dilatation $\mu(z)$ for which in turn, by the Measurable Riemman mapping theorem, see, e.g. [1], [2, p. 67], the Beltrami equation (1.5) generates a quasiconfomal homeomorphism $\omega: \Omega \rightarrow G$. As the domain $G$ one can take any plane domain which is conformally equivalent to $\Omega$. It is well 
known that $\omega \in W_{\text {loc }}^{1, p}(\Omega), 2 \leq p<\frac{2 K}{K-1}, \omega \in C_{\mathrm{loc}}^{\alpha}(\Omega), \alpha=1 / K$, and we see that if $\omega(z)=u(z)+i v(z)$, then $\operatorname{div}(A(z) \nabla u)=0, \operatorname{div}(A(z) \nabla v)=0$, a.e. in $\Omega$ and $u, v \in W_{\text {loc }}^{1,2}(\Omega)$. In what follows we will say that the matrix $A$ generates the corresponding quasiconformal mapping $\omega$, or $A$ and $\omega$ are agreed.

Remark 1. If we replace the uniform ellipticity condition (1.7) by the weaker inequality of the ellipticity $|\mu(z)|<1$ a.e. in $\Omega$, then, under some additional assumptions with respect to $\mu(z)$ that guarantee the existence of a homeomorphic solution $\omega(z)=u(z)+i v(z) \in W_{\text {loc }}^{1,1}(\Omega)$ to the degenerate Beltrami equation, one can repeat the above considerations. The existence problem for the degenerate Beltrami equation, i.e., when

$$
K(z)=\frac{1+|\mu(z)|}{1-|\mu(z)|} \notin L^{\infty},
$$

is currently an active area of research; see, e.g., the recent books $[2,12]$, which contain, in particular, the extended bibliography on the topic.

In this paper we study the blow-up problem for a model semilinear equation $\operatorname{div}(A(z) \nabla u)=e^{u}$ in $\Omega$ and show that the well-known LiouvilleBieberbach function solves the problem under an appropriate choice of the matrix $A(z)$. The proof is based on the fact that every regular solution $u$ can be expressed as $u(z)=T(\omega(z))$ where $\omega: \Omega \rightarrow G$ stands for quasiconformal homeomorphism generated by the matrix $A(z)$ and $T$ is a solution of the semilinear weihted Bieberbach equation $\triangle T=m(w) e^{T}$ in $G$. Here the weight $m(w)$ is the Jacobian determinant of the inverse mapping $\omega^{-1}(w)$.

\section{A reducing lemma}

Let $\Omega$ be a domain in the complex plane $\mathbb{C}$. In what follows, we will use for the sake of simplicity in the exposition the symmetric matrices written in the form

$$
A=\left(\begin{array}{cc}
\alpha & \beta \\
\beta & \delta
\end{array}\right)
$$

with smooth entries $\alpha, \beta, \delta,|\mu(z)|<1$, with the exception of a finite number of points in $\Omega$, and such that $\operatorname{det} A=1$.

Lemma 1. Let $\omega: \Omega \rightarrow G$ be a homeomorphic solution to the Beltrami equation (1.5) agreed with the matrix $A, T$ be a real valued function in $C^{2}(G)$ and $u=T \circ \omega$. Then

$$
\operatorname{div}[A(z) \nabla u(z)]=J_{\omega}(z) \triangle T(w), w=\omega(z),
$$

where $J_{\omega}(z)$ stands for the Jacobian determinant of the mapping $\omega(z)$. 
Proof. Assuming that $T \in C^{2}(G)$ and that $\omega(z)=a(x, y)+i b(x, y)$, $z=x+i y$, is a homeomorphic solution to the Beltrami equation (1.5), we see that in $\Omega$

$$
\begin{gathered}
\left\{\begin{array}{c}
b_{y} \quad=\alpha a_{x}+\beta a_{y} \\
-b_{x}=\beta a_{x}+\delta a_{y},
\end{array}\right. \\
\alpha \delta-\beta^{2}=1,
\end{gathered}
$$

and

$$
\begin{cases}-a_{y} & =\alpha b_{x}+\beta b_{y} \\ a_{x} & =\beta b_{x}+\delta b_{y} .\end{cases}
$$

Setting $u(z)=T(\omega(z))$, we get

$$
\nabla u(z)=D^{t}(\omega) \nabla T(w), w=\omega(z),
$$

and therefore

$$
\begin{aligned}
\operatorname{div}[ & A(x, y) \nabla u(x, y))]=\frac{\partial}{\partial x}\left[\alpha\left(T_{\xi} a_{x}+T_{\eta} b_{x}\right)\right. \\
& \left.+\beta\left(T_{\xi} a_{y}+T_{\eta} b_{y}\right)\right]+\frac{\partial}{\partial y}\left[\beta\left(T_{\xi} a_{x}+T_{\eta} b_{x}\right)+\delta\left(T_{\xi} a_{y}+T_{\eta} b_{y}\right)\right] \\
& \left.\left.=T_{\xi} \operatorname{div}[A(x, y) \nabla a(x, y))\right]+T_{\eta} \operatorname{div}[A(x, y) \nabla b(x, y))\right]+Q(x, y),
\end{aligned}
$$

where

$$
\begin{aligned}
& Q(x, y)=T_{\xi \xi}\left(\alpha a_{x}^{2}+2 \beta a_{x} a_{y}+\delta a_{y}^{2}\right)+ \\
& \quad T_{\eta \eta}\left(\alpha b_{x}^{2}+2 \beta b_{x} a_{y}+\delta b_{y}^{2}\right)+2 T_{\eta \xi}\left(\alpha a_{x} b_{x}+\delta a_{y} b_{y}+\beta a_{y} b_{x}+\beta a_{x} b_{y}\right) .
\end{aligned}
$$

Since

$$
\operatorname{div}[A(x, y) \nabla a(x, y)]=\frac{\partial}{\partial x}\left[\alpha a_{x}+\beta a_{y}\right]+\frac{\partial}{\partial y}\left[\beta a_{x}+\delta a_{y}\right]=0
$$

and

$$
\operatorname{div}[A(x, y) \nabla b(x, y)]=\frac{\partial}{\partial x}\left[\alpha b_{x}+\beta b_{y}\right]+\frac{\partial}{\partial y}\left[\beta b_{x}+\delta b_{y}\right]=0,
$$

we have that

$$
\operatorname{div}[A(x, y) \nabla u(x, y))]=Q(x, y) .
$$

By (2.3),

$$
\left(\alpha a_{x} b_{x}+\delta a_{y} b_{y}+\beta a_{y} b_{x}+\beta a_{x} b_{y}\right)=0
$$

and

$$
\left(\alpha a_{x}^{2}+2 \beta a_{x} a_{y}+\delta a_{y}^{2}\right)=\left(\alpha b_{x}^{2}+2 \beta b_{x} a_{y}+\delta b_{y}^{2}\right)=a_{x} b_{y}-a_{y} b_{x}=J_{\omega}(z) .
$$


Hence,

$$
Q(x, y)=J_{\omega}(z) \triangle T(w), w=\omega(z), \text { a.e in } \Omega
$$

and we arrive at the formula (2.2).

Remark 1. If $A$ is the unit matrix, then we arrive at the well-known result for the Laplace equation: if $T$ is harmonic and $\omega$ is analytic, then $\triangle(T(\omega(z)))=\left|\omega^{\prime}(z)\right|^{2} \triangle T(\omega(z))$, and therefore $T(\omega(z))$ is also harmonic function. Next, if $u$ is an $A$-harmonic function, then $T$ is harmonic.

\section{Semilinear elliptic equations}

Let $\Omega$ be a bounded domain in $\mathbb{C}$ and let $\partial \Omega$ denote its boundary. In this section we deal with the semilinear equation

$$
\operatorname{div}[A(z) \nabla u(z)]=f(u(z))
$$

and the boundary condition

$$
u(z) \rightarrow+\infty \text { as } d(z):=\operatorname{dist}(z, \partial \Omega) \rightarrow 0,
$$

as well as with its Laplace's counterpart

$$
\begin{gathered}
\triangle u(z)=f(u(z)), \\
u(z) \rightarrow+\infty \text { as } d(z):=\operatorname{dist}(z, \partial \Omega) \rightarrow 0 .
\end{gathered}
$$

Solutions to these problems are called boundary blow-up solutions, or large solutions.

In the last decades semilinear equations became a central subject of study in the theory of nonlinear partial differential equations. The study of such equations is of interest because of its numerous applications to actual problems of differential geometry, mathematical physics, logistic problems etc.: see, e.g., $[7,8]$, and the extended bibliography therein.

The existence of a large solution to the equation (3.3) is related to the existence of a maximal solution $u$ of (3.3) in $\Omega$, which in turn depends on the so called Keller-Osserman condition, see $[16,18]$. J.B. Keller and R. Osserman provided a sharp condition on the growth of $f$ at the infinity which guarantees that the set of solutions (3.3) is uniformly bounded from above in compact subsets of $\Omega$. Qualitatively the condition means that the superlinearity of $f$ at the infinity is sufficiently strong. They derived an a priory estimate for solutions of (3.3) in terms of $\rho(z)=\operatorname{dist}(z, \partial \Omega)$. This estimate implies that the equation (3.3), in bounded domain, possesses a maximal solution. Under some additional conditions on $\Omega$ the maximal 
solution blows up everywhere on the boundary, i.e one arrives at a large solution.

Recall that a function $f \in C\left(\mathbb{R}_{+}\right)$satisfies the Keler-Osserman condition if there exists a positive non-decreasing function $h$ such that

$$
f(t) \geq h(t), \forall t \in \mathbb{R}_{+} \text {and } \int_{t_{0}}^{\infty}\left\{\int_{0}^{t} h(s) d s\right\}^{-1 / 2} d t<\infty \text { for all } t_{0}>0 .
$$

It is known that if $f$ is non-decreasing and satisfies the Keller-Osserman condition, then a large solution exists in every bounded smooth domain. Uniqueness in smooth domains was established under some additional conditions on $f$, see e.g. [17], Section 5.3. It is easy to check that the functions $f(t)=e^{t}$ and $f(t)=t^{p}, p>1$, satisfy (3.5). The semilinear equation

$$
\triangle u=e^{u},
$$

as far as we know, was first investigated by Bieberbach in his pioneering work [4] related to the study of Riemannian geometry and automorphic functions in the plane. More precisely, if a Riemannian metric of the form $|d s|^{2}=e^{2 u(x)}|d x|^{2}$ has constant Gaussian curvature $-g^{2}$, then $\triangle u=$ $g^{2} e^{2 u}$. It is this work that has stimulated numerous studies in the field of semilinear differential equations in $R^{n}, n \geq 1$, and the equation (3.6) continues to play the role of one of the fundamental model equations of the theory. It is important to note that in simply connected planar domains $\Omega$ the large solutions for the equation (3.6) are expressed explicitly by means of the Liouville-Bieberbach formula

$$
u(z)=\log \frac{8\left|f^{\prime}(z)\right|^{2}}{\left(1-|f(z)|^{2}\right)^{2}}
$$

where $f$ stands for a conformal map $f: \Omega \rightarrow \mathbb{D}=\{z:|z|<1\}$.

For the model case of the equation

$$
\Delta u=e^{a u}, a>0,
$$

the following result holds, see [17], Theorem 5.3.7.

Theorem A. Let $\Omega$ be a bounded domain in $\mathbb{C}$ such that $\partial \Omega=\partial \bar{\Omega}^{c}$. Then there exists one and only one blow-up solution to (3.8).

\section{Some Applications of Lemma 1}

Below we confine ourselves to a few examples of the application of Lemma 1 to the study of some properties of boundary blow-up solutions of a classical model semilinear elliptic equation $\operatorname{div}[A(z) \nabla u]=p(z) e^{u}$. 
Theorem 1. Let $k(t), 0<t<1$, be an arbitrary complex valued smooth function, such that $|k(t)| \leq q<1$ and the matrix $A$ is generated by the Beltrami coefficient $\mu(z)=k(|z|) z / \bar{z}$ in accordance with formula (1.8). Then the boundary blow-up solution to the equation

$$
\operatorname{div}[A(z) \nabla u(z)]=p(|z|) e^{u(z)}
$$

in the unit disk $\mathbb{D}$ where

$$
p(t)=\frac{1-|k(t)|^{2}}{|1-k(t)|^{2}} \exp \left\{\int_{1}^{t} \operatorname{Re} \frac{4 k(\tau)}{1-k(\tau)} \frac{d t \tau}{\tau}\right\}
$$

has the explicit representation

$$
u(z)=\log \frac{8}{\left(1-|\omega|^{2}\right)^{2}} .
$$

Here

$$
|\omega|=\exp \left\{\int_{1}^{|z|} \operatorname{Re} \frac{1+k(t)}{1-k(t)} \frac{d t}{t}\right\} .
$$

Proof. By the Measurable Riemann mapping theorem, there exists unique normalized quasiconformal self-homeomorphism $\omega(z)$ of the unit disk $\mathbb{D}$ with the Beltrami coefficient $\mu(z)=k(|z|) z / \bar{z}$ and this homeomorphism is written explicitly, see [2, p. 82],

$$
\omega(z)=\frac{z}{|z|} \exp \left\{\int_{1}^{|z|} \frac{1+k(t)}{1-k(t)} \frac{d t}{t}\right\} .
$$

We see that

$$
\begin{aligned}
& \frac{\omega_{\bar{z}}}{\omega}=\frac{k}{1-k} \frac{1}{\bar{z}}, \\
& \frac{\omega_{z}}{\omega}=\frac{1}{1-k} \frac{1}{z}
\end{aligned}
$$

and therefore, for the Jacobian $J_{\omega}(z)=\left|\omega_{z}\right|^{2}-\left|\omega_{\bar{z}}\right|^{2}$, we have that

$$
J_{\omega}(z)=\frac{1-|k|^{2}}{|1-k|^{2}} \frac{|\omega|^{2}}{|z|^{2}}=p(|z|) .
$$

We look for a solution to the equation (4.1) in the form $u=T \circ \omega$, assuming that $T \in C^{2}(\mathbb{D})$. Making use of Lemma 1 , we see that $T$ satisfies the Bieberbach equation

$$
\triangle T=e^{T} \text { in } \mathbb{D} .
$$


By the mentioned above Bieberbach's result [4] and referring also to Theorem A, we see that the unique $C^{2}(\mathbb{D})$ boundary blow-up solution to the equation (4.3) in the unit disk $\mathbb{D}$ is given by the explicit formula

$$
T(w)=\log \frac{8}{\left(1-|w|^{2}\right)^{2}} .
$$

Therefore, the required solution to the equation (4.1) has the explicit representation

$$
u(z)=\log \frac{8}{\left(1-|\omega(z)|^{2}\right)^{2}},
$$

where $\omega(z)$ is given by formula (4.2).

Among the quasiconformal automorphisms (4.2) of the unit disc $\mathbb{D}$ there is a variety of volume-preserving maps, for which $J_{\omega}(z) \equiv 1, z \in \mathbb{D}$. The following statement may have of independent interest.

Corollary 1.1. Let $\mathbb{D}$ be the unit disk in the complex plane $\mathbb{C}$ centered at the origin and let the matrix $A(z)$ be generated by the Beltrami coefficient

$$
\mu(z)=k(|z|) \frac{z}{\bar{z}}
$$

in accordance with formula (1.8) where

$$
k(t)=\nu^{2}(t) \pm i \nu(t) \sqrt{1-\nu^{2}(t)}
$$

and $\nu(t), 0<t<1$, stands for an arbitrary real-valued smooth function, such that $|\nu(t)| \leq q<1$. Then there exists one and only one boundary blow-up solution to the classical semilinear equation

$$
\operatorname{div}[A(z) \nabla u]=e^{u}, \quad z \in \mathbb{D},
$$

which is written explicitly by the Liouville-Bieberbach formula

$$
u(z)=\log \frac{8}{\left(1-|z|^{2}\right)^{2}} .
$$

Proof. Let $A(z)$ be a matrix generated by the complex Beltrami coefficient (4.6), satisfying (4.7). In order to apply Theorem 1, we have to verify that $J_{\omega}(z) \equiv 1$ and $|\omega(z)|=|z|$ for $z \in \mathbb{D}$. Indeed, in our case $\operatorname{Re} k=|k|^{2}$ and therefore $|\omega(z)|=|z|, z \in \mathbb{D}$. The later implies that $J_{\omega}(z) \equiv 1$. 
For example, the well-known spiral mapping

$$
s(z)=z e^{2 i \log |z|}
$$

is just the volume preserving. Indeed, in this case the Beltrami coefficient

$$
\mu(z)=\frac{\omega_{\bar{z}}}{\omega_{z}}=\frac{1}{2}(1+i) \frac{z}{\bar{z}}
$$

and we see, that it corresponds to (4.7) with $\nu(t) \equiv 1 / \sqrt{2}$. If $z=\rho e^{i \varphi}$. The matrix $A$ generated by such $\mu(z)$ will depend on $\varphi$ only and has the form

$$
A=\left(\begin{array}{cc}
3-2(\cos 2 \varphi-\sin 2 \varphi) & -2(\cos 2 \varphi+\sin 2 \varphi) \\
-2(\cos 2 \varphi+\sin 2 \varphi) & 3+2(\cos 2 \varphi-\sin 2 \varphi)
\end{array}\right) .
$$

The spiral mappings play an important role in applications. F. Gehring employed $s(z)$ in [9] to solve the well-known Bers's problem on the structure of the universal Teichmüller space. F. John [14] used the mapping $s(z)$ to study the uniqueness of non-linear elastic equilibrium for prescribed boundary displacements. The spiral mappings also play an important role in the theory of smooth planar mappings with constant principal stretches, see $[6,10]$. The problem of conformal differentiation as well as many regularity, distortion and rotation problems for quasiconformal mappings in the plane were also investigated by means of the spiral mapping. We recall a typical such problem that went back to F. John which closely related to the non-linear elasticity theory. In [13] he showed that if $f: \mathbb{C} \rightarrow \mathbb{C}$ is an $(1+\varepsilon)$ - bilipschitz mapping and if for some $0<a<b$ we have $f(z)=z$ for $|z|>b, f(z)=z e^{i \theta}$ for $|z|<a$, then

$$
|\theta| \leq C(1+\log (b / a)) \varepsilon .
$$

The angle estimate (4.11) follows from the basic stability theorems in [13] for $(1+\varepsilon)$-bilipschitz mappings in the plane. The BMO technique [15] also plays an important role for (4.11). Quasiconformal methods lead to the sharp solution of John's problem, see [11], [2, Chapter 13]. It is shown that the logorithmic spiral gives the extremum to F. John's angle distortion problem for plane bilipschitz mappings.

Theorem 2. Let $\Omega$ be the annulus $r<|z|<1$ in the complex plane $\mathbb{C}$ and let the matrix $A(z)$ be generated by the Beltrami coefficient

$$
\mu(z)=k(|z|) \frac{z}{\bar{z}}
$$


in accordance with formula (1.8) where

$$
k(t)=\nu^{2}(t) \pm i \nu(t) \sqrt{1-\nu^{2}(t)}
$$

and $\nu(t), 0<t<1$ stands for an arbitrary real-valued smooth function. If $|\nu(t)| \leq q<1$, then there exists one and only one boundary blow-up solution to the classical model semilinear equation

$$
\operatorname{div}[A(z) \nabla u]=e^{u} \text {, in the annulus } r<|z|<1,
$$

which is written explicitly by the formula

$$
u(z)=\log \frac{2 \pi^{2}}{|z|^{2}\left(\log ^{2} r\right) \cdot \sin ^{2}\left(\frac{\pi}{\log r} \log |z|\right) .}
$$

Proof. Arguing in the same way as in the proof of Theorem 1, we see that $u(z)=T(\omega(z)), r<|z|<1$, where quasiconformal self-homeomorphism $\omega(z)$ of the annulus is given by (4.2) and $T$ is the boundary blow-up solution to the semilinear equation

$$
\triangle T=e^{T}
$$

in the annulus $r<|w|<1$. By Bieberbach's result [4] and Theorem A the unique boundary blow-up solution to the equation (4.16) is given by the formula

$$
T(\omega)=\log \frac{8\left|F^{\prime}(\omega)\right|^{2}}{\left(1-|F(\omega)|^{2}\right)^{2}}
$$

where $F(\omega)$ stands for a conformal mapping of the annulus $r<|\omega|<$ 1 onto the unit disk. It remains to find the corresponding conformal mapping $F(w)$. We see that: 1) $w=\log \omega$ maps the annulus onto the strip $\log r<\operatorname{Re} w<0 ; 2) \zeta=-i e^{\frac{\pi}{\log r} i w}$ maps the strip onto the right half-plane; 3) $t=(\zeta-1) /(\zeta+1)$ maps the right half-plane onto the unit disk. Composing the above mappings we get the required formula

$$
F(\omega)=\frac{i e^{\frac{\pi}{\log r} i \log \omega}+1}{i e^{\frac{\pi}{\log r} i \log \omega}-1} .
$$

Next, setting $\tau(\omega)=i e^{\frac{\pi}{\log r} i \log \omega}$, we see that

$$
\left(1-|F(\omega)|^{2}\right)^{2}=\frac{16 \operatorname{Re}^{2} \tau(\omega)}{|\tau-1|^{4}}
$$

where

$$
\operatorname{Re} \tau(\omega)=-|\tau| \sin \left(\frac{\pi}{\log r} \log |\omega|\right) .
$$


On the other hand,

$$
\left|F^{\prime}(\omega)\right|^{2}=\frac{4}{|\tau-1|^{4}}\left|\tau^{\prime}(\omega)\right|^{2}=\frac{4}{|\tau-1|^{4}}|\tau|^{2} \frac{\pi^{2}}{\log ^{2} r} \frac{1}{|\omega|^{2}}
$$

Thus

$$
T(\omega)=\log \frac{2 \pi^{2}}{|\omega|^{2}\left(\log ^{2} r\right) \cdot \sin ^{2}\left(\frac{\pi}{\log r} \log |\omega|\right)}
$$

and since $|\omega(z)|=|z|$, we complete the proof of Theorem 2 .

Making use of the limit in (4.15) as $r \rightarrow 0$ we get the following result which may have of independent interest.

Corollary 2.1 The semilinear equation

$$
\operatorname{div}[A(z) \nabla u]=e^{u}
$$

for each matrix A from Theorem 2, as well as the Bieberbach equation

$$
\triangle u=e^{u}
$$

admit the following boundary blow-up solution in the punctured unit disk $0<|z|<1$

$$
u(z)=\log \frac{2}{|z|^{2} \log ^{2}|z|}
$$

Remark 1. The approach given above to the construction of a boundary blow-up solution to the Bieberbach equation in the unit disk $\mathbb{D}$ with a singularity at the origin can be extended to the case of finite number of singular points $z_{k},\left|z_{k}\right|<1, k=1,2, \ldots, n$. Indeed, let $r>0$ be such that all the circles $d_{k}=\left\{z:\left|z-z_{k}\right| \leq r\right\}$ belong to $\mathbb{D}$ and do not intersect each other. Denote by $F_{r}(z)$ a conformal mapping of the circular multi-connected domain $\mathbb{D} \backslash \cup_{k=1}^{n} d_{k}$ onto the unit disc $\mathbb{D}$. Then the required solution with prescribed singularities at the points $z_{k}$ is given by

$$
u(z)=\lim _{r \rightarrow 0} \log \frac{8\left|F_{r}^{\prime}(z)\right|^{2}}{\left(1-\left|F_{r}(z)\right|^{2}\right)^{2}} .
$$

Remark 2. We have considered the uniformly elliptic case only, i.e. when $|\nu(|z|)| \leq q<1$. It can be shown that the above formulae remain hold also in the case when $|\nu(|z|)|<1$ a.e., assuming the convergence of the corresponding integrals. 
Now we show how to produce a boundary blow-up solution to the equation $\operatorname{div}[A(z) \nabla u(z)]=e^{u}$ in the punctured disk $0<|z|<1$, making use of the cavitation effect and the degeneration of the matrix $A$.

Theorem 3. Let the matrix $A(z)$ be generated by the Beltrami coefficient

$$
\mu(z)=-\frac{1}{1+2|z|} \frac{z}{\bar{z}} .
$$

Then there exists a boundary blow-up solution to the degenerate elliptic semilinear equation

$$
\operatorname{div}[A(z) \nabla u(z)]=e^{u}
$$

in the punctured disk $\mathbb{D} \backslash\{0\}$, such that $u(z) \rightarrow+\infty$ as $|z| \rightarrow 1$ and $z \rightarrow 0$. Moreover,

$$
u(z)=T\left(z\left(1+\frac{1}{|z|}\right)\right)
$$

where $T$ stands for a boundary blow-up solution to the equation

$$
\triangle T(w)=\left(1-\frac{1}{|w|}\right) e^{T}
$$

in the annulus $1<|w|<2$.

Proof. In spite of the fact that $|\mu(z)| \rightarrow 1$ as $z \rightarrow 0$, the Beltrami coefficient (4.26) generates unique normalized homeomorphisms to the degenerate Beltrami equation

$$
\omega(z)=(1+1 /|z|) z, \omega(1)=2,
$$

that maps $\mathbb{D} \backslash\{0\}$ onto the annulus $1<|w|<2$. Since $\omega_{\bar{z}}=-z / 2 \bar{z}|z|$, $\omega_{z}=1+1 / 2|z|$, we see that the Jacobian of the mapping $\omega$ is written as

$$
J_{\omega}(z)=1+\frac{1}{|z|} .
$$

Because the inverse mapping

$$
z=\omega^{-1}(w)=(R-1) e^{i \tau}, w=R e^{i \tau},
$$

we see that

$$
J_{\omega^{-1}}(w)=1-\frac{1}{|w|} .
$$

If $u(z)$ is a solution of the degenerate elliptic equation (4.27) in the punctured unit disk $\mathbb{D}$ and $u(z)=T(\omega(z))$, then

$$
T(w)=u \circ \omega^{-1}(w)
$$


solves the equation

$$
\triangle T(w)=\left(1-\frac{1}{|w|}\right) e^{T}
$$

in the annulus $1<|w|<2$. Since the weight

$$
\left(1-\frac{1}{|w|}\right)>0
$$

in the annulus $1<|w|<2$, we conclude that there exists a boundary blow-up solution to (4.31) in the annulus $1<|w|<2$, i.e. $T(w) \rightarrow \infty$ as $|w| \rightarrow 1$ and $|w| \rightarrow 2$. Thus, $u(z)=T(\omega(z))$ with $\omega(z)$ of the form (4.30) is just the required solution. Here we have used of the following remark from [3]: It is not difficult to see that the Keller-Osserman condition for the equation $\triangle u(x)=m(x) f(u(x))$ still holds even in the case where $m(x)$ vanishes at the boundary $\partial \Omega$, provided it is positive in a neighborhood of $\partial \Omega$.

We complete the set of examples given above by an explicit solution with singularity for a degenerate semilinear elliptic equation in the punctured unit disk $\mathbb{D} \backslash\{0\}$.

Theorem 4. Let the matrix $A(z)$ is generated by the Beltrami coefficient

$$
\mu(z)=-\frac{1}{1+2|z|} \frac{z}{\bar{z}} .
$$

Then a boundary blow-up solution to the problem

$$
\operatorname{div}[A(z) \nabla u(z)]=\left(1+\frac{1}{|z|}\right) e^{u(z)}, \quad z \in \mathbb{D} \backslash\{0\},
$$

with singularity at the origin is written explicitly

$$
u(z)=\log \frac{8 \pi^{2}}{(1+|z|)^{2}\left(\log ^{2} 2\right) \cdot \sin ^{2}\left(\frac{\pi}{\log 2} \log (1+|z|)\right)} .
$$

Proof. In spite of the fact that $|\mu(z)| \rightarrow 1$ as $z \rightarrow 0$, the degenerate Beltrami equation (1.5) admits unique normalized homeomorphism in the punctured unit disk $\mathbb{D} \backslash\{0\}$, which is given by the formula (4.13):

$$
\omega(z)=\frac{1+|z|}{|z|} z .
$$


This function $\omega(z)$ maps $\mathbb{D} \backslash\{0\}$ onto the annulus $1<|\omega|<2$ and its Jacobian $J_{\omega}(z)=(1+1 /|z|)$. Looking the solution as $u(z)=T(\omega(z))$, we see, by Lemma 1 , that $T$ satisfies the Bieberbach equation

$$
\triangle T=e^{T}
$$

in the annulus $1<|w|<2$, the boundary blow-up solution of which is

$$
T(w)=\log \frac{8 \pi^{2}}{|w|^{2}\left(\log ^{2} 2\right) \cdot \sin ^{2}\left(\frac{\pi}{\log 2} \log |w|\right)},
$$

see formula (4.21) with $r=2$. Thus, the desired solution

$$
u(z)=T(\omega(z))
$$

and since $|\omega(z)|=1+|z|$, we complete the proof.

Remark 3. Perhaps a reader has pointed out that Lemma 1 is proved for matrices $A$ with smooth coefficient. It is done only for the sake of simplicity in the exposition. We plan to publish the relevant results for the matrices with measurable coefficients, satisfying the uniform ellipticity condition, as well as to study the case of degeneration.

\section{References}

[1] L. V. Ahlfors, Lectures on Quasiconformal Mappings, Princeton, N.J.: Van Nostrand, 1966; Reprinted by Wadsworth Ink. Belmont, 1987.

[2] K. Astala, T. Iwaniec, G. Martin, Elliptic partial differential equations and quasiconformal mappings in the plane, Princeton Math. Ser. 48, Princeton University Press, Princeton, NJ, 2009.

[3] C. Bandle, Yu. Cheng, G. Porru, Boundary blow-up in semilinear elliptic problems with singular weights at the boundary // Institut Mitag-Leffler, Report No. 39, (1999/2000), 1-14.

[4] L. Bieberbach, $\triangle u=e^{u}$ und die automorphen Funktionen // Math. Ann., 7 (1916), No. 7, 173-212.

[5] B. Bojarski, V. Gutlyanskii, O. Martio, V. Ryazanov, Infinitesimal geometry of quasiconformal and bi-lipschitz mappings in the plane, EMS Tracts in Mathematics, 19, European Mathematical Society (EMS), Zurich, 2013, 205 p.

[6] M. Chuaqui, J. Gevirtz, Constant principal strain mappings on 2-manifolds // SIAM J. Math. Anal., 32 (2000), 734-759.

[7] J. I. Diaz, Nonlinear partial differential equations and free boundaries. Vol. I. Elliptic equations, Research Notes in Mathematics, 106, Pitman (Advanced Publishing Program), Boston, MA, 1985, 323 p. 
[8] M. Ghergu, V. Radulescu, Nonlinear PDEs. Mathematical models in biology, chemistry and population genetics, Springer Monographs in Mathematics, Springer, Heidelberg, 2012, 391 p.

[9] F. W. Gehring, Spirals and the universal Teichmüller space // Acta Math., 141 (1978), 99-113.

[10] J. Gevirtz, On planar mappings with prescribed principal strains // Arch. Rational Mech. Anal., 117 (1992), 295-320.

[11] V. Gutlyanskiu,, O. Martio, Rotation estimates and spirals // Conform. Geom. Dyn., 5 (2001), 6-20.

[12] V. Gutlyanskii, V. Ryazanov, U. Srebro, E. Yakubov, The Beltrami equation. A geometric approach, Dev. Math. 26, Springer-Verlag, New York, 2012, 301 p.

[13] J. John, Rotation and strain // Comm. Pure Appl. Math., 14 (1961), 391-413.

[14] F. John, Uniqueness of non-linear elastic equilibrium for prescribed boundary displacements and sufficiently small strains // Comm. Pure Appl. Math., 25 (1972), 617-634.

[15] F. John, L. Nirenberg, On functions of bounded mean oscillation // Comm. Pure Appl. Math., 14 (1961), 415-426.

[16] J. B. Keller, On solutions of $\triangle u=f(u) / /$ Comm. Pure Appl. Math., 10 (1957), 503-510.

[17] M. Marcus, L. Veron, Nonlinear second oder elliptic equations involving measures, De Gruyter, Series in nonlinear analysis and applications, 21, Berlin/Boston, 2014, 248 p.

[18] R. Osserman, On the inequality $\triangle u \geq f(u)$ // Pacific J. Math., 7 (1957), 16411647.

\section{CONTACT INFORMATION}

\section{Vladimir}

Gutlyanskiü,

Olga Nesmelova, Vladimir Ryazanov
Institute of Applied Mathematics

and Mechanics,

National Academy of Sciences of Ukraine,

Ukraine, Slavyansk

E-Mail: vladimirgut@mail.ru,

star-o@ukr.net,

vl_ryazanov@mail.ru 